\title{
LÄNDLICHE R̈̈UME IN LATEINAMERIKA: GLOBALISIERUNG UND LOKALE REAKTIONEN
}

\section{ESPAÇOS RURAIS NA AMÉRICA LATINA: REAÇÕES GLOBAIS E LOCAIS}

\author{
Martina Neuburger \\ Universität Hamburg - Hamburg - Germany
}

\begin{abstract}
Kurzfassung: Die ländlichen Räume in Südamerika unterliegen in den letzten Jahrzehnten zunehmenden Globalisierungseinfl üssen, die zu einem tiefgreifenden Strukturwandel geführt haben, der bis heute im Gange ist. Dabei sind nicht nur Veränderungen in den wirtschaftlichen Bereichen zu beobachten. Auch sozio-kulturelle, politische und ökologische Sphären werden restrukturiert. Die damit verbundenen Prozesse erfassen somit praktisch alle Lebensbereiche der ländlichen Bevölkerung, so dass Akteure auf lokaler und regionaler Ebene gezwungen sind, sich neu zu positionieren. Die Reaktionen reichen dabei von der aktiven Anpassung an und Integration in globalisierte Wirkungszusammenhänge bis hin zum Rückzug aus den übergeordneten Kontexten und der Erschließung neuer Handlungsoptionen auf lokaler Ebene. Diese vielfältigen Prozesse in den ländlichen Räumen Südamerikas lassen sich in verschiedene Entwicklungsdynamiken diff erenzieren. Die agro-industrielle Entwicklungsdynamik ist charakterisiert durch eine Landwirtschaft, in der standardisierte Massenprodukte dominieren, die von Billiglohnarbeitern hergestellt werden und für den Massenkonsum vor allem auf globaler Ebene bestimmt sind. In der postproduktivistischen Entwicklungsdynamik fungiert der ländliche Raum hingegen als Konsumraum einer städtischen oder/und globalen Elite, die ihre Freizeit dort gestalten will und ökologische Ausgleichsfl ächen für die „eigene“ degradierte Umwelt benötigt. In der als alternativ bezeichneten Entwicklungsdynamik schließlich dominieren eindeutig die lokal-regionalen Akteure, deren Hauptaugenmerk auf der Überlebenssicherung der lokalen Bevölkerung, auf der Sicherung der ökologischen Ressourcen und auf der Erhaltung der lokalen Gemeinschaft liegt. Diese Entwicklungsdynamiken überlagern sich zeitlich und räumlich. Die vielschichtigen kleinräumigen Diff erenzierungen sind verbunden mit einer entsprechenden Erweiterung der Funktionen der ländlichen Räume im lokalregionalen, nationalen und internationalen Kontext. Ländliche Entwicklungsplanung und die darin involvierten politischen Entscheidungsträger stehen damit vor neuartigen Herausforderungen, die neue Konzepte und Leitbilder verlangen.
\end{abstract}

\begin{abstract}
In recent decades, rural regions in South America have increasingly been infl uenced by global impacts that, until today, result in profound structural transformations. Th ese globalizing processes restructure economic fi elds as well as socio-cultural, political and ecological spheres. Th ereby, the rural population in South America suff ers modifi cations in all areas of everyday life. Actors on the local and regional level are therefore forced to re-position themselves within this new framework. Th e reactions range from an active adaptation and integration into globalized networks, to the retraction of all higherlevel contexts and the development of new perspectives on local level. We can characterize these multifaceted processes in rural spaces of South America by three diff erent types of dynamics. Within the agro-industrial dynamic global players govern largescale agriculture of standardized products, employing basically low-wage workers and supplying mass consumer markets. Within the post-productivist dynamic, rural regions serve as space to be "consumed" by urban or global elites who are searching for personal leisure and a compensation of their "own" degraded spaces. And fi nally, within the alternative dynamic, local and regional actors clearly dominate all processes. Th eir main objective is to ensure the survival of the local population and the environmental resources as well as to sustain the local community. Th ese three types of dynamics superimpose each other and result in manifold small-scale diff erences in socioeconomic and political processes. Th ereby, rural planning and political decision makers are confronted with new challenges that demand for new concepts and visions for rural spaces.
\end{abstract}

\section{Einführung}


Die ländlichen Räume Südamerikas unterliegen seit vielen Jahrzehnten - mit geringfügigen Unterschieden von Land zu Land - intensiven Globalisierungseinfl üssen. Basis dafür waren und sind umfangreiche politische Maßnahmen, die die Agrarmärkte liberalisierten, räumliche Planung und öff entliche Dienstleistungen auch und gerade im ländlichen Raum auf ein Minimum reduzierten bzw. tertiärisierten und nicht-staatlichen Akteuren vielfach „das Feld“ überließen. Diese politischen Rahmenbedingungen bildeten die Voraussetzungen für die direkte Wirkung der Globalisierungseinfl üsse auf regionaler und lokaler Ebene. Dabei lassen sich die Eff ekte sowohl in wirtschaftlichen, als auch in sozio-kulturellen, politischen und ökologischen Sphären erkennen (siehe Abb. 1).

Abb. 1. Globalisierungseff ekte in ländlichen Räumen Lateinamerikas

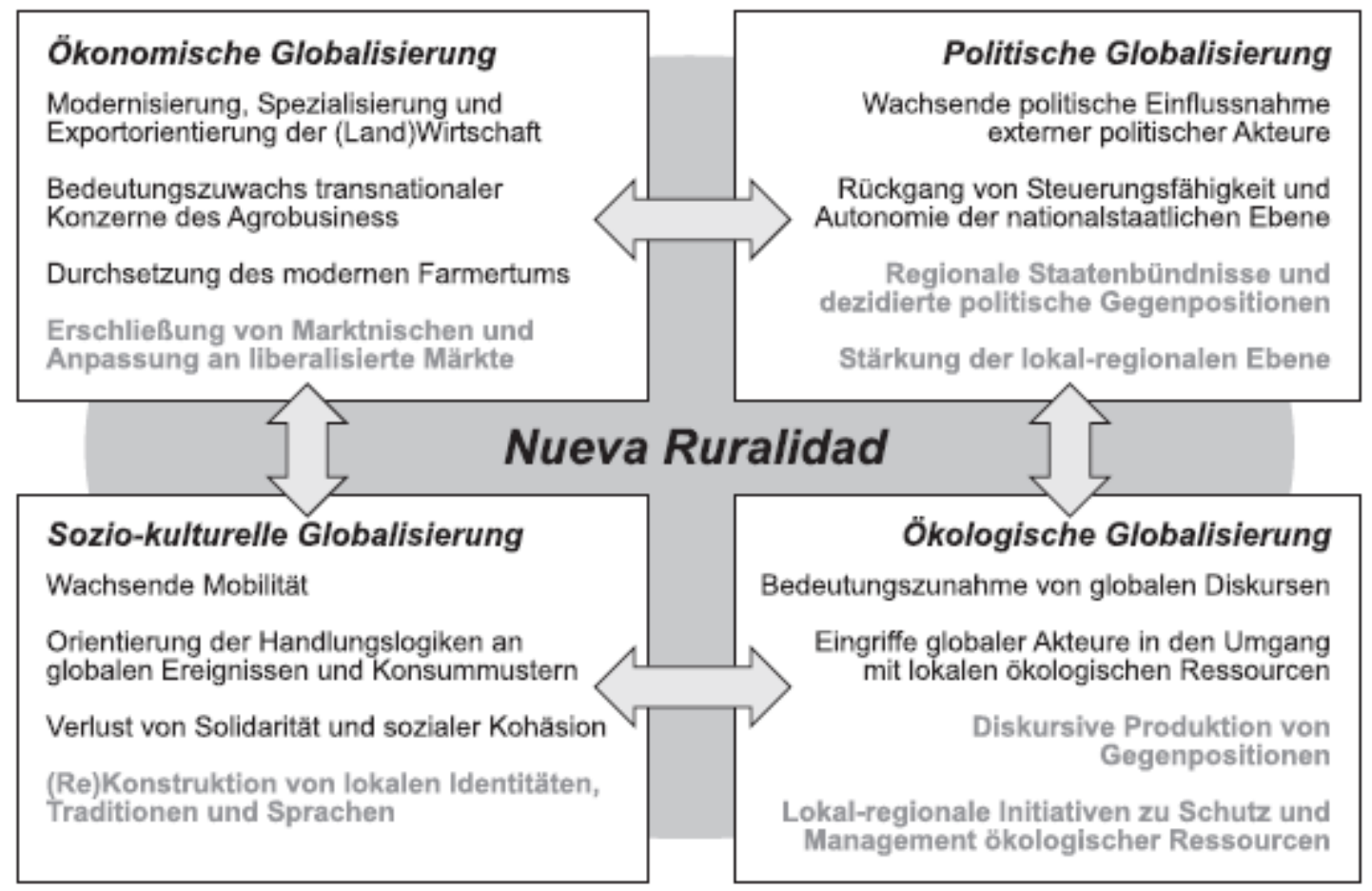

Entwurf: Martina Neuburger 2008.

Allerdings beschränken sie sich nicht ausschließlich auf die Wirkung globaler Entwicklungen auf die untergeordneten Ebenen, die davon mehr oder weniger passiv betroff en sind. Vielmehr handeln lokale und regionale Akteure - im Sinne des globallocal-interplay-pro-aktiv im Rahmen der neuen Chancen, die sich aus der Globalisierung für sie ergeben. Im Folgenden werden die Globalisierungseff ekte in ländlichen Räumen ateinamerikas in ihren unterschiedlichen Facetten dargestellt. Dabei fokussiert der Beitrag zunächst auf die ökonomischen Einfl üsse der Globalisierung, die zweifellos weitreichende Folgen auch für die anderen Lebensbereiche der ländlichen Bevölkerung haben. Daran anschließend werden die sozio-kulturellen Dynamiken beleuchtet, die durch zunehmende globale Einfl üsse in den lateinamerikanischen Gesellschaften beobachtbar sind. In einem dritten Teil steht die Globalisierung der politischen Macht im Vordergrund der Analyse. Schließlich werden die Wirkungen der Globalisierung auf die Naturaneignung näher untersucht. In allen Kapiteln werden dabei sowohl die Globalisierungseinfl üsse dargelegt, als auch die lokalen Reaktionen und Gegenbewegungen erläutert. Im Schlusskapitel werden diese vier Stränge der Globalisierungseff ekte zusammengeführt und systematisiert. 


\section{Globalisierung ländlicher Ökonomien in Lateinamerika}

Im Zuge der Öff nung der Märkte sowie mit der weltweiten Beschleunigung von Waren-, Kapital- und Informationsströmen sind die ländlichen Räume Lateinamerikas einer globalen Konkurrenz ausgesetzt. Als Standorte für die landwirtschaftliche Produktion von Lebens- und Futtermitteln sowie Rohstoff en können zahlreiche lateinamerikanische Regionen durch niedrige Bodenpreise und billige Arbeitskräfte bei gleichzeitig relativ hohen technologischen Standards und einem guten Ausbildungsniveau konkurrenzfähig arbeiten. In den entsprechenden GewinnerRegionen modernisiert und spezialisiert sich die Landwirtschaft, setzt neueste Technologien und importiertes Know-How ein und orientiert die Produktion auf den Weltmarkt. Die entsprechenden Wertschöpfungsketten werden im Wesentlichen von transnationalen Konzernen - vor allem Monsanto und Cargill - kontrolliert. Die Konzerne engagieren sich jedoch selten selbst in der Produktion, sondern binden die Produzenten über Kredit- und Lieferverträge an sich und verlagern damit das Produktionsrisiko auf die landwirtschaftlichen Betriebe. Nur vereinzelt im Bereich der high value products - vor allem in der Wein-, Gemüse- und Obstproduktion - steigen Agroindustrie-Unternehmen in die Produktion ein, um ausreichende Liefermengen in der geforderten Qualität zu garantieren (World Bank 2007).

Durch die Globalisierung der Landwirtschaft entstehen Inseln der in globale Wirtschaftskreisläufe integrierten Bereiche, die sich regional sehr unterschiedlich manife- stieren. Der Anbau von Soja, der vor allem in Brasilien und Argentinien seit den 1970er Jahren an Bedeutung zugenommen hat, konzentriert sich auf den Süden und Mittelwesten Brasiliens, auf den Oriente Boliviens sowie auf die Chacogebiete Paraguays und Argentiniens, expandiert jedoch insgesamt nach Norden und Westen ( Neuburger 2008a). Die auf den Weltmarkt ausgerichteten Intensivkulturen Wein, Obst und Gemüse haben sich vor allem in den Bewässerungsoasen der Küstenwüsten Perus, des Andenfußes Chiles und Argentiniens sowie am Rio São Francisco etabliert (Voth 2002, Marshall 2009). Gewächshauskulturen für Schnittblumen sind vorwiegend in Kolumbien und Ecuador zu fi nden, während Fleisch im Wesentlichen aus der argentinischen Pampa, teilweise auch aus Südbrasilien und Uruguay exportiert wird. Tabak gehört zu den wichtigsten Exportkulturen in Südbrasilien, während in Kuba diese Produktion nach wie vor staatlicher Kontrolle unterliegt (Karnopp 2006). Durch die internationalen Diskussionen und neuen Abkommen zu Agrotreibstoff en wie Rizinus, Sonnenblumen und Zuckerrohr ist zu erwarten, dass auch diese Kulturen in den nächsten Jahren sehr stark an Dynamik zunehmen und ihre Anbaufl ächen expandieren (Kohlhepp 2008, Hees et al. 2007). Zahlreiche Alkoholdestillerien in Brasilien wurden bereits von internationalen Investorengruppen und Hedgefonds aufgekauft, um die zu erwartenden steigenden Renditen für ihre Kunden zu sichern.

Für die Regionen bedeutet die Expansion solcher globalisierter Produktionsformen,

dass einerseits die Beanspruchung des Bodens und damit die Erosionsgefahr wächst. Grundsätzlich haben in sozialer Hinsicht die Intensivkulturen sehr viel größere Beschäftigungseff ekte als die stark mechanisierten bzw. mechanisierbaren Anbauprodukte Soja, Zuckerrohr oder Getreide. Unabhängig davon werden ressourcenarme Kleinbauern aus solchen Regionen verdrängt, da sie nicht das notwendige Kapital haben, um ihre Produktion entsprechend zu modernisieren. Häufi g werden sie auch von ihrem Land vertrieben, wenn sie keinen formalisierten Landtitel besitzen und die modernisierten Betriebe ihr Land wissentlich oder unwissentlich vom 
Staat oder anderen im Kataster eingetragenen Besitzern kaufen oder pachten, um ihre Anbaufl ächen auszudehnen (Jacobson 2005, Reboratti 2005).

Gegen diese Verdrängungsprozesse, die durch die Globalisierung erneut an Dynamik gewonnen haben, wehren sich die betroff enen Bevölkerungsgruppen der ländlichen Räume. Etwas besser gestellte Kleinbauern mit einem gewissen Kapitalstock und frei verfügbaren Ressourcen versuchen, sich an die Anforderungen der liberalisierten Märkte anzupassen. Sie erschließen durch die Spezialisierung ihrer Produktion sehr spezifi sche Marktnischen, die für Großbetriebe nicht zugänglich sind. Meist handelt es sich dabei um high value products höchster Qualität, bei denen vor allem Sorgfalt und Handarbeit notwendig sind oder der persönliche Kontakt zum Kunden von entscheidender Bedeutung ist. Obst und Gemüse gehören zu denjenigen Produkten, die vor allem für kleinbäuerliche Betriebe auch auf dem Weltmarkt - meist jedoch über den transnationalen Handel - Nischen bieten. Für Ökoprodukte oder andere Lebensmittel, die eine vermarktbare regionale Herkunft aufweisen oder mit besonders traditionellen Methoden gefertigt wurden, für die es jedoch in lateinamerikanischen Ländern noch keine allgemein anerkannte Zertifi zierung gibt, bildet vor allem der direkte vertrauensvolle Kundenkontakt die Basis für ihre Vermarktung auf regionaler Ebene. Um in der globalen und regionalen Konkurrenz $\mathrm{zu}$ bestehen, sind unternehmerisches Denken und Risikobereitschaft bzw. ausreichend verfügbare Ressourcen, die eine solche zulassen, Grundvoraussetzungen für den Erfolg kleinerer und mittlerer Betriebe.

Neben diesen relativ reichen Betrieben haben auch die ressourcenarmen Familien Strategien entwickelt, um sich gegen Verdrängung und Proletarisierung zu wehren. Die landlos gewordenen Kleinbauern schließen sich zunehmend zu neuen sozialen Bewegungen zusammen und haben sich inzwischen weltweit über die Vía Campesina vernetzt. Zweifellos am erfolgreichsten sind in diesem Kontext die Zapatistas im Süden Mexikos und die Landlosenbewegung (Movimento dos Trabalhadores Rurais sem Terra) in Brasilien (Mançano Fernandes 2008, Moyo \& Yeros 2005, Gabbert 2004, Branford \& Rocha 2002).

Neben der Landwirtschaft setzen sich auch in den anderen Wirtschaftssektoren, die für den ländlichen Raum in Lateinamerika relevant sind, zunehmend globalisierte Produktionsformen durch. Im Tourismus locken vor allem schöne Strände und imposante Landschaften internationale Investoren an. Sie investieren vorwiegend in große Hotelanlagen, in denen Touristen in geschlossenen Resorts all-inclusive riesige Strände, luxuriöse Freizeit- und Vergnügungseinrichtungen, teilweise auch Golfplätze ungestört genießen können. Eine der größten internationalen Hotelketten im oberen Preissegment der Drei- bis Fünf-Sterne-Hotels, die außer in Europa vor allem in Lateinamerika Niederlassungen hat, ist Sol Meliá mit Sitz auf Mallorca, mit weltweit 328 Hotels in 27 Ländern und circa 32500 Beschäftigten (Sol Meliá 2005). Allein in Lateinamerika gehören 58 Hotelanlagen zu Sol Meliá, 34 davon sind Ressorts, von denen wiederum 20 in Kuba und 8 in Mexiko liegen. Diese Anlagen, die ausschließlich für Gäste und Personal zugänglich sind, besetzen die schönsten Landschaften, so dass für kleinere Tourismusunternehmen mit geringeren Investitionsvolumina oder für lokale Interessenten nur sekundäre Zielgebiete bleiben. Durch die enge Verfl echtung von globalen Reiseveranstaltern, Fluggesellschaften und Hotelketten konzentrieren sich die internationalen Kunden gerade im Hochpreissegment des Tourismus fast ausschließlich auf die entsprechenden Einrichtungen, so dass diese Bereiche mit den größten Gewinnspannen den Großinvestoren vorbehalten bleiben.

Der Bergbau, als weiterer wichtiger Wirtschaftsfaktor im ländlichen Raum, gewinnt in Lateinamerika ebenfalls zunehmend an Bedeutung. Aufgrund der 
wachsenden Rohstoff nachfrage auf dem Weltmarkt, der Privatisierung staatlicher Bergbaugesellschaften und der Vergabe von Abbaukonzessionen konnten in den letzten Jahren international Investoren in diesem Bereich Fuß fassen (Coy \& Töpfer 2009, Svampa \& Antonelli 2009). Dabei handelt es sich meist um Konsortien mehrerer nationaler und internationaler Unternehmen in Zusammenarbeit mit (halb-)staatlichen Bergbaufi rmen, die an einem Großprojekt beteiligt sind. Die weltweit größten Bergbaukonzerne Vale, Rio Tinto und BHP Billiton sind entsprechend in mehreren Abbaugebieten in Lateinamerika vertreten (siehe Abb. 2).

\section{$A b b .2$ : Bergbau in Lateinamerika}

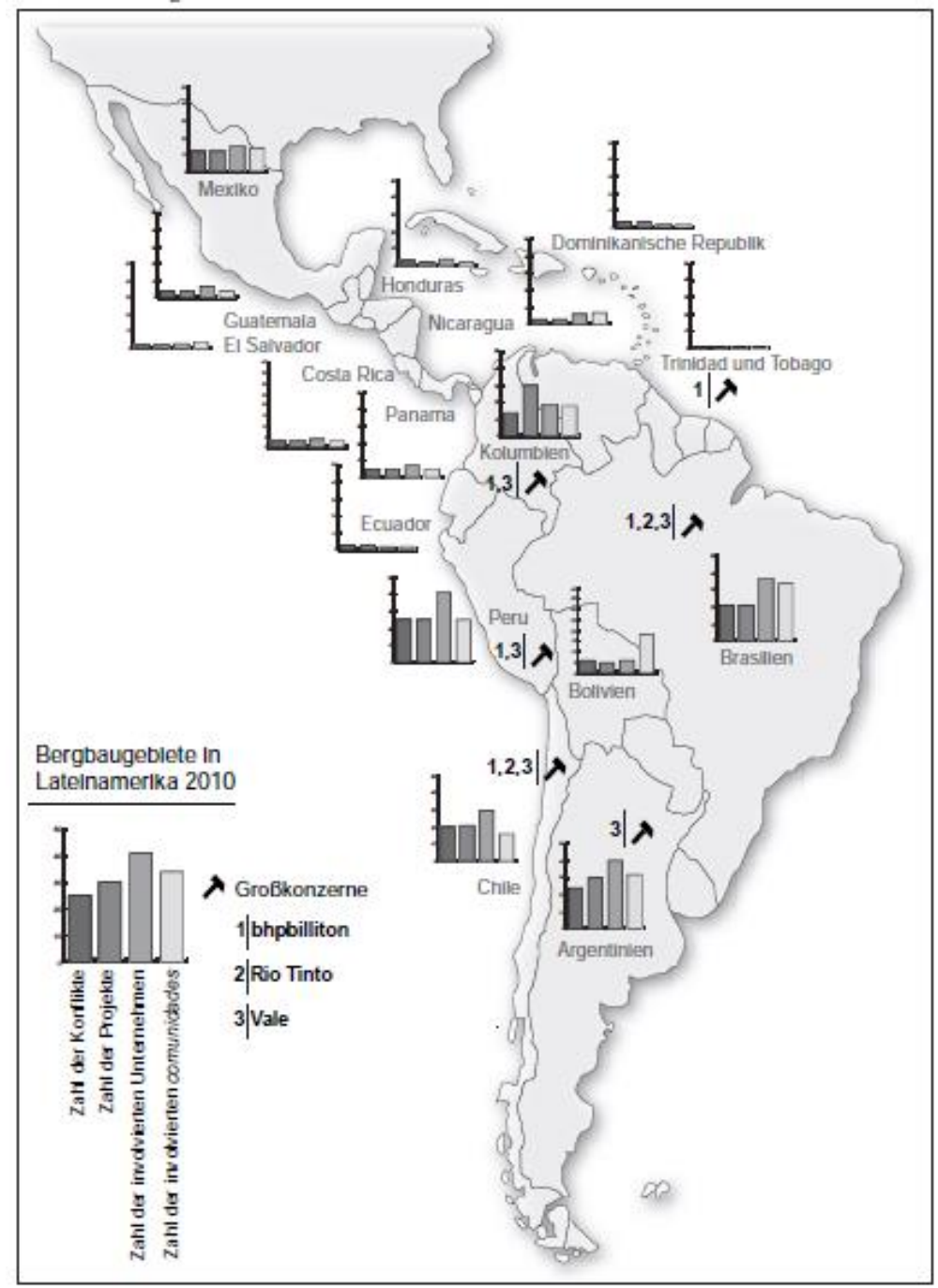

Quelle: bhpbilliton 2009, Rio Tinto 2010, Vale 2008, OLCA 2010

Entwurf: Martina Neuburger 2010 Kartographie: Silke Greth.

In den direkt angrenzenden Gebieten sind aufgrund des hohen Mechanisierungsund Automatisierungsgrades nur sehr geringe Regionalentwicklungseff ekte des Bergbaus zu erkennen, auch wenn die Unternehmen - teilweise über die Bezahlung von vertraglich festgesetzten royalties - Projekte zur sozialen und wirtschaftlichen Entwicklung in der Region durchführen, die dann in den jeweiligen Nachhaltigkeitsberichten der Unternehmen ausführlich dargestellt werden. Die geringen Regionalentwicklungseff ekte verbunden mit den weit verbreiteten, teilweise auch politisch hochgepushten negativen ökologischen Auswirkungen haben in den letzten 
Jahren dazu geführt, dass immer heftigere Konfl ikte um die Ausbeutung von Lagerstätten entstehen. Den Protesten der lokalen Bevölkerung schließen sich Solidaritätsbewegungen nicht nur aus dem ganzen Land, sondern in ganz Lateinamerika an und unterstützen die Demonstrationen logistisch und personell. Über moderne Kommunikationsmedien sind die unterschiedlichen Gruppierungen miteinander vernetzt, tauschen rasch Informationen aus, ziehen Experten zur Beratung heran und suchen nach politischer Unterstützung. Eine der größten und bestorganisierten Bewegungen ist die No a la Mina (übersetzt: „Nein zur Mine”, siehe www.noalamina.org). In ihr haben sich die so genannten vecinos autoconvocados (eigenorganisierte Nachbarschaften) zusammengeschlossen, organisieren regelmäßig Treff en auf regionaler, nationaler und gesamt-lateinamerikanischer Ebene und koordinieren Protestaktionen. Es handelt sich dabei um eine sehr dynamische und $\mathrm{fl}$ exible Bewegung, die keine eindeutigen Führungspersönlichkeiten hat, deren Aktivisten sich aus sehr heterogenen Gruppen zusammensetzen, die sich kontinuierlich ändern, und deren politische Schwerpunkte entsprechend häufi $\mathrm{g}$ wechseln. Dennoch ist die Stoßrichtung der Bewegung - ähnlich wie in anderen Widerstandsbewegungen - gegen den transnationalen Bergbau, der als Ausverkauf der volkseigenen Ressourcen gesehen wird, gegen ökologische Zerstörung und gegen Intransparenz gerichtet. Inzwischen hat No a la Mina politisch ein vergleichsweise großes Gewicht, das Bergbauprojekte zumindest zeitweise stoppen konnte und Regierungen zwingt, zu ihnen Position zu beziehen und Abbaukonzessionen öff entlich zu rechtfertigen.

\section{Sozio-kulturelle Globalisierungseff ekte in den lateinamerikanischen Gesellschaften}

In den lateinamerikanischen Gesellschaften äußert sich die zunehmende Globalisierung vor allem in der wachsenden Mobilität über nationalstaatliche Grenzen hinweg. Migration bildet zwar schon seit vielen Generationen eine der wichtigsten Überlebensstrategien im ländlichen Raum lebender Familien, jedoch haben sich die Migrationsmuster in den letzten Jahrzehnten insgesamt sehr stark gewandelt: Während noch in den 1970er Jahren die Etappenwanderung vom ländlichen Raum über Kleinund Mittelstädte bis hin zu den Metropolen dominierte und entsprechend die Vergroßstädterung vorantrieb, verschieben sich die Migrationsströme seit den 1980er Jahren starker hin zu den Mittelstädten, so dass diese die höchsten Wachstumsraten aufweisen. Seit den 1980er Jahren nimmt gleichzeitig die Zahl der grenzüberschreitenden Migranten kontinuierlich zu. Im Jahr 2000 waren es bereits über 21 Millionen in Lateinamerika geborene Personen, die im Ausland lebten, was knapp 4 \% der Gesamtbevölkerung Lateinamerikas entspricht (Solimano \& Allendes 2007: 16). Eindeutiges Hauptziel der Migration sind schon seit vielen Jahrzehnten die USA, wo 18 Millionen Lateinamerikaner leben. Seit Mitte der 1990er Jahre gewinnt jedoch Spanien als Wanderungsziel an Bedeutung. Gleichzeitig hat auch die innerlateinamerikanische Migration zugenommen (Rodríguez Vignoli 2004). So wandern zum Beispiel vor allem Bolivianer nach Argentinien, Peruaner nach Chile, Nicaraguaner nach Costa Rica und Haitianer in die Dominikanische Republik. Dabei haben sich die Ursachen der Migration nicht grundsätzlich geändert: große Einkommensunterschiede zwischen Herkunftsland und Zielgebiet, wirtschaftliche Instabilität und Krisen, Arbeitslosigkeit und Informalität, politische Repression und interne Konfl ikte. Die abwandernden Gruppen haben sich jedoch verschoben hin zu mehr Frauen, Personen mit zunehmend guter Ausbildung und im produktivsten Alter (UN 2009).

Mit dem Anwachsen der internationalen Migration gewinnen die Remissen immer 
mehr an Bedeutung, da die Einkommensunterschiede meist sehr viel größer sind als bei der früheren Migration innerhalb eines Landes (Adams Jr. \& Page 2005). Darüber hinaus sind diese Geldtransfers rasch und kostengünstig erst mit der Liberalisierung der Finanzmärkte und der Einführung neuer Informationstechnologien möglich. In den letzten Jahren haben diese Zahlungen als Devisenbringer für die lateinamerikanischen Volkswirtschaften einen wachsenden Stellenwert mit mehr als 62 Millionen US\$ im Jahr 2006. Im Jahr 2002 erreichten sie in vergleichsweise kleinen Staaten wie Haiti, Nicaragua und Jamaica erwartungsgemäß weit mehr als $20 \%$ des Bruttoilandprodukts, wobei die Werte seitdem weiterhin stark gestiegen sind. Aber auch in wirtschaftsstarken Staaten wie Mexiko, Kolumbien und Brasilien haben die Remissen einen Anteil am BIP von $3 \%, 2 \%$ und $1 \%$ (Solimano \& Allendes 2007: 39ff ). Für die einzelnen Familien bedeutet der Erhalt von Remissen eine Vervielfachung des monatlichen Einkommens, das sie dann in Lebenshaltungskosten, in Neubau oder Verbesserung von Wohngebäuden, in Ausbildung, in Aufbau bzw. Ausbau von Unternehmen oder in den Erwerb von Immobilien investieren. Die Regierungen der Herkunftsländer hoff en deshalb auf Regionalentwicklungseff ekte und fördern entsprechende Migrantenvereine im Ausland, um so die Bindung der Ausgewanderten an die Heimat zu stärken und ihre Rückkehr zu unterstützen (Vono de Vilhena 2006).

Die Migration über nationalstaatliche Grenzen hinweg hat sich nicht zuletzt deswegen beschleunigt, weil gerade die Mobilität von relativ ressourcenarmen Bevölkerungsgruppen aus Gründen der Risikominimierung sehr stark von bestehenden Kontakten an den Zielorten abhängen. Durch die Privatisierung der lateinamerikanischen Telefongesellschaften in den 1990er Jahren und die damit verbundene Verbreitung moderner Kommunikationsmedien im ländlichen Raum hat sich die Vernetzung zwischen Herkunfts- und Zielgebiet der Migranten enorm verbessert. Dies ist insbesondere dort von großer Bedeutung, wo sich die Migranten im illegalen oder semilegalen Bereich bewegen. Dies wird beispielsweise deutlich bei den bolivianischen Einwanderern in Spanien, die nur mit Hilfe der bereits in Spanien mit legalem Status wohnenden Bolivianer die hohen bürokratischen Hürden umgehen, als Touristen einreisen und danach von den Behörden unbehelligt dort leben und arbeiten können (Jerger 2008, Husa et al. 2000).

Positiv wie negativ tragen die ethnischen und familiären Netzwerke zur Reproduktion von Migration bei. Die weltweiten sozialen Verfl echtungen werden dadurch dichter und schaff en neue Identitäten zwischen Herkunfts- und Zielgebieten der Migranten. Diese weltweite Kommunikation - unterstützt durch Fernsehen und Internet - verbreiten globalisierte Konsummuster und Lebensstile. Lokale Traditionen verlieren an Bedeutung, auch weil in Gebieten mit hoher Abwanderungsrate nur Rumpff amilien zurück bleiben, die solche Riten und Feste nicht allein ausrichten können. Darüber hinaus kann eine sehr ungleiche Verteilung von Remissen in ländlichen comunidades Neid fördern, so dass die Solidarität sich immer stärker an den weltweiten Netzwerken orientiert und nicht mehr an die lokal verortete Gemeinschaft gebunden ist. Diese zunehmende Orientierung an globalen Zusammenhängen wird noch verstärkt durch die nahezu ubiquitäre Verfügbarkeit von Informationen über Ereignisse an anderen Standorten weltweit. Handlungslogiken richten sich dadurch zunehmend an global

kommunizierten Idealen, Werten und Ängsten aus. Die Aufnahme des weltweiten Diskurses um den Klimawandel in die Alltagsgespräche der ländlichen Bevölkerung zeigt diese Wirkungen sehr deutlich.

Gegen die zunehmende Außenorientierung von Wertesystemen und Netzwerken setzen zahlreiche Bevölkerungsgruppen im ländlichen Raum Lateinamerikas auf die 
Stärkung lokaler Zusammenhänge. Insbesondere die indígenas nutzen die Wiederbelebung oder Neuerfi ndung von Identität und Traditionen in ihrer lokalen Verankerung, um Territorien und Ressourcen für sich zu sichern (Cleary \& Steigenga 2004, Bayardo \& Lacarrieu 2003). Zur Legitimierung ihrer Ansprüche überführen sie gewissermaßen ihre gelebten, erzählten und tradierten kulturellen Praktiken wie Sprache, Ethnowissen und Mythen in die formalisierten Systeme des dominanten Staates, indem sie sie verschriftlichen, markieren, vermessen und dokumentieren. Auch in ländlichen comunidades, die nicht auf indigene Wurzeln zurückgreifen können, wachsen die Bemühungen um die Pfl ege von lokalen Traditionen und Identitäten, selbst wenn die Siedlungen - wie in Kolonisationsgebieten - nur wenige Jahrzehnte alt sind. Die Gründung von Folklore-Gruppen, die Organisation von Kunsthandwerkmärkten und die Veranstaltung von Kochwettbewerben dienen insbesondere in ländlichen Abwanderungsgebieten dazu, das Selbstbewusstsein zu stärken. Teilweise entstanden solche lokalen Initiativen aus den comunidades heraus. Vielfach wurden sie jedoch vor allem in den 1990er Jahren von staatlichen Stellen und über Regionalförderprogramme gepusht, um - so das damals und zum Teil noch heute herrschende Paradigma - das endogene Potenzial und die nachhaltige Entwicklung zu stärken (Beispiele aus Brasilien siehe Kohlhepp 1998). Auch wenn die Maßnahmen vielfach ins Leere liefen und mit der nicht aufzuhaltenden Entleerung des ländlichen Raumes keine Basis mehr hatten, so brachten sie in einzelnen Munizipien empowerment-Prozesse in Gang, die zur Verbesserung der Lebensbedingungen im lokalen Kontext führten (siehe beispielsweise Neuburger 2006).

Die politischen Dimensionen lokaler Vernetzung zeigen sich auch in anderen Bereichen. Lokale Radiosender haben bereits eine lange Tradition in Lateinamerika und warden zum Informationsaustausch über lokal und regional relevante Ereignisse genutzt.

Insbesondere in Konfl iktsituationen hatte und hat dieses technisch sehr einfache Kommunikationsmedium große Bedeutung wie beispielsweise bei den Protesten in Oaxaca im Jahr 2006, bei denen eine zunächst friedliche Demonstration der Lehrergewerkschaft vom Militär brutal niedergeschlagen wurde (Dehn 2009, Budka 2004, Hoff -mann \& Schulz 2002). Auch bei den Konfl ikten um den transnationalen Bergbau oder bei Landkonfl ikten und drohender Gewalt durch Polizei, Großgrundbesitzer oder Bergbaugesellschaft gegenüber Landlosen, Kleinbauern oder indígenas konnten und können Radiosender erfolgreich eingesetzt werden.

\section{Die Globalisierung politischer Macht: Die Entmachtung des Nationalstaates}

In den 1980er und 1990er Jahren haben die lateinamerikanischen Staaten die Staatsquote in der Wirtschaft entscheidend gesenkt und sich damit aus der Steuerung wirtschaftlicher Prozesse stark zurückgezogen. Häufi g unter Androhung empfi ndlicher Sanktionen oder mit dem Knebel der Kreditverweigerung beeinfl ussen supranationale Institutionen wie Welthandelsorganisation, Weltbank und Internationaler Währungsfonds die nationalen Politiken. Um diesen Tendenzen entgegen zu wirken, versuchten die lateinamerikanischen Staaten schon Anfang der 1990er Jahre, regionale Bündnisse zu stärken. Insbesondere die Gründung des MERCOSUR 1991 sollte, dem Beispiel Europas folgend, die regionale Integration vorantreiben und so durch koordinierte Politiken vor allem im wirtschaftlichen Bereich sowie durch das gemeinsame Auftreten auf internationaler Bühne den beteiligten Einzelstaaten nach innen wie nach außen mehr Gewicht geben. Allerdings konnten diese Bemühungen nur sehr begrenzte Erfolge verzeichnen, da durch vergleichsweise ähnliche 
Wirtschaftsstrukturen gegenseitige Konkurrenzen um dieselben Märkte entstanden. Darüber hinaus fürchteten die kleineren Mitgliedstaaten Uruguay, Paraguay und das assoziierte Bolivien, dass Brasilien seine Vormachtstellung in der Region ausbauen wollte, und verhielten sich deshalb sehr zögerlich bei der Unterzeichnung gemeinsamer Handelsabkommen (Calcagnotto \& Nolte 2000). Auch andere Bündnisse wie der Amazonas- oder der Andenpakt blieben bislang ohne große Wirkungen, da es viele Staaten vorziehen, mit ihren jeweils wichtigsten Handelspartnern - vor allem mit den USA - Einzelabkommen abzuschließen, in denen ihre spezifi schen Interessen stärker einbezogen wurden.

Parallel dazu wächst mit dem neuen Jahrtausend das Selbstbewusstsein der lateinamerikanischen Nationalstaaten, die nach einer Phase der wirtschaftlichen Stagnation und der politischen Dauerkrise wieder eine gewisse Stabilität genießen. Dies ist zum einen sicherlich mit der wirtschaftlichen und weltpolitischen Krise der USA verbunden, die mit wachsender Verschuldung und zunehmenden Armutsphänomenen zu kämpfen haben und durch ihr imperialistisches Auftreten in der internationalen Terrorbekämpfung an politischem Ansehen verloren haben. Zum anderen haben die Regierungen Lateinamerikas durch ihre ähnlichen politischen linksorientierten Ausrichtungen in entscheidenden Fragen trotz großer Rivalitäten untereinander einen Schulterschluss geschaff $\mathrm{t}$, der ihre Positionierung gegenüber den USA und anderen Industrienationen stärkt (Stolowicz 2008).

Mit unterschiedlichen Akzenten fördern sie nationalistische Interessen und kehren teilweise wie in Bolivien oder Venezuela wieder zur Verstaatlichung zentraler Wirtschaftssektoren wie Bergbau und Energie zurück, um die Macht transnationaler Konzerne im eigenen Land $\mathrm{zu}$ brechen (Coy \& Töpfer 2009). Auch führen die Regierungen Sozialprogramme ein und nehmen damit die Strukturanpassungsmaßnahmen der 1990er Jahren zumindest zum Teil wieder zurück (Lander 2008, Moldiz Mercado 2008). In Venezuela werden - aus den staatlichen Einnahmen des Erdölexports fi nanziert - monatliche Stipendien für Schüler und Studierende ausbezahlt, landwirtschaftliche Kooperativen gegründet und die Monopolstellung der Einzelhandelskonzerne im Rahmen der Misión Alimentación aufgehoben. In Brasilien sollen Programme wie Fome Zero, Bolsa Família, Programa Nacional de Fortalecimento da Agricultura Familiar und Luz para Todos die Infrastruktur im ländlichen Raum verbessern und die Armut bekämpfen (Hall 2006, Guanziroli 2007). In Bolivien investiert der Staat in den Ausbau von Gesundheits- und Bildungsinfrastruktur in ländlichen Regionen und beschleunigt die Agrarreform durch die Überarbeitung des Landreform-Gesetzes Ley INRA (INRA 2010). Einen wichtigen Impuls gegen zunehmende globale Einfl üsse auf die politischen Schwerpunkte bildeten die vielfältigen Gesetze zur Dezentralisierung von Verwaltung und politischen Entscheidungskompetenzen. Die bekanntesten Beispiele sind die partizipative Haushaltsplanung in zahlreichen Munizipien und Städten Brasiliens und das Gesetz der Participación Popular in Bolivien (siehe der Beitrag in diesem Band von Rodrigues Mororó und Nijenhuis 2010). Allerdings besteht das Problem in der Umsetzung der Programme vor allem in ländlich-peripheren Regionen darin, dass die lokalen Akteure logistisch und personell überfordert sind, so dass sich häufi g bereits bestehende lokale Machtverhältnisse in den entsprechenden Entscheidungsgremien reproduzieren und die ungleiche Machtverteilung noch perpetuieren (zum Beispiel Bolivien siehe Neuburger 2010).

\section{Die Globalisierung der Naturaneignung}


Die Nutzung und Aneignung von Natur ist in Lateinamerika spätestens seit den 1980er Jahren von globalen Einfl üssen geprägt. Wechselnde ineinander übergehende Diskurse über Waldschutz, Klimawandel und Biodiversität legitimierten den Zugriff externer Akteure auf die amazonischen Wälder in sehr unterschiedlichen Formen (Simonis 1996). Gegen den direkten Eingriff ausländischer Akteure wehrte sich vor allem Brasilien, das bis heute hinter diesem großen Engagement der Industrieländer für den Waldschutz eine Strategie zur Internationalisierung Amazoniens vermutet. Deshalb werden in den letzten Jahren vor allem Maßnahmen diskutiert, die im Sinne von ecossystem services die jeweiligen Staaten, regionale und vor allem lokale Nutzer der Wälder für potenzielle Einnahmeeinbußen im Falle der Schonung der ökologischen

Ressourcen entschädigen soll. Das erst vor Kurzem verabschiedete REDD-Abkom men im Rahmen der Kyoto-Nachverhandlungen zielt in eine ähnliche Richtung (Parker et al. 2009, Schmidt 2009).

Diese meist diskursive Aneignung der tropischen Regenwälder durch globale Akteure zeigt auf regionaler und lokaler Ebene sehr widersprüchliche Wirkungen. Bei der Ausweisung von Schutzgebieten kam es abhängig vom zugrundeliegenden Schutzkonzept regelmäßig zu Konfl ikten um die Nutzungsrechte (Röper 2001, Redford \& Fearn 2007). Lokale Bevölkerungsgruppen, die im Schutzgebiet selbst lebten oder am Rande davon, wurden in ihren Nutzungsformen eingeschränkt oder umgesiedelt bzw. aus ihren Siedlungsgebieten verdrängt. Andere Akteure versuchten, sich Entschädigungen $\mathrm{zu}$ erschleichen, indem sie sich kurz vor der Ausweisung solcher Gebiete dort ansiedelten. Teilweise war die Durchsetzungsfähigkeit von Waldschutzmaßnahmen sehr gering, wenn massive wirtschaftliche Interessen dem entgegenstanden. Deshalb überlagern sich heute Schutzgebiete mit Holzeinschlagskonzessionen oder Erdöl- und Gasfördergenehmigungen. Abhängig davon, welche Akteure erfolgreicher in der politischen Lobbyarbeit auch über die nationalstaatlichen Grenzen hinaus sind, setzen sich die Schutz- oder die Extraktionsinteressen durch (Neuburger 2007 und 2008b).

Gegen die wachsende Einfl ussnahme von globalen Akteuren auf die Ressourcennutzung wehren sich nationale wie regional-lokale Akteure gleichermaßen. Die beständigen Forderungen, die im Rahmen der Klimadiskussionen von internationalen Entwicklungsagenturen vor allem an die Staaten mit Regenwaldanteil herangetragen werden, empfi nden die lateinamerikanischen Regierungen vielfach als Bevormundung. Entsprechend reagieren sie und bauen Gegendiskurse auf, die den internationalen Interessen die Legitimation entziehen. Sie beschuldigen die Industriestaaten beispielsweise des ökologischen Imperialismus, der lediglich ein Vehikel sei, um die Hegemonie des Nordens gegenüber dem Süden zu sichern. Neue gesetzliche Regelungen,besonders in den Amazonasstaaten, sichern inzwischen vor allem das vielfältig vorhandene Ethnowissen und garantieren den indigenen Gruppen die Bezahlung von royalties bei der wirtschaftlichen Inwertsetzung ihres Wissens.

Auch auf regionaler und lokaler Ebene sind in Lateinamerika inzwischen zahlreiche Initiativen zu beobachten, die sich um den Umwelt- und Naturschutz im eigenen Umfeld bemühen. Die Ausweisung von kommunalen Schutzgebieten und das Management von Flusseinzugsgebieten sind nur einige der wenigen Maßnahmen, die von Gemeinden und lokalen Umweltbewegungen getragen und häufi g von staatlichen Stellen unterstützt werden. Lokale Initiativen setzen sich vor allem dort für den Schutz des regionalen Ökosystems ein, wo ihr Überleben damit unmittelbar zusammenhängt. Eines der prominentesten Beispiele dürfte der Kampf der Kautschukzapfer im brasilianischen Bundesstaat Acre sein, bei dem ihr Anführer Chico Mendes von gegnerischen Großgrundbesitzern, die ihre Weidefl ächen ausdehnen wollten, 
umgebracht wurde (Neuburger \& Coy 2002). Neben den indigenen Gruppen, die um die Sicherung ihrer Territorien auch und gerade in Zusammenarbeit mit globalen Umweltschutz- und Menschenrechtsbewegungen kämpfen, sind es zunehmend auch andere Bevölkerungsgruppen, die sich aktiv gegen die Zerstörung ihrer Lebensräume zur Wehr setzen. Lokale Fischerkooperativen in Amazonien beispielsweise wehren sich gegen die kommerzielle Fischerei, die zunehmend ihre Fischbestände bedroht (Hoefl e 2004). Diese lokalen Initiativen, die meist auf partizipativen Entscheidungen basieren, halten den globalen Konzepten von Wald- und Klimaschutz Strategien entgegen, die an die jeweiligen spezifi schen Rahmenbedingungen und die Bedürfnisse der einheimischen Bevölkerung angepasst sind und so ein Gegengewicht dazu darstellen.

\section{Fazit: Entwicklungsdynamiken und Funktionen ländlicher Räume im Globalisierungskontext Lateinamerikas}

Die dargelegten vielfältigen Globalisierungseinfl üsse und die lokal-regionalen Reaktionen darauf spiegeln unterschiedliche Entwicklungsdynamiken wider, die den ländlichen Räumen spezifi sche Funktionen zuweisen und - in Anlehnung an ein Modell von Marsden (2003) - bestimmten Regulationsmechanismen folgen (siehe Abb. 3). Dabei defi nieren sich die Entwicklungsdynamiken aus den dominanten bzw. Involvierten Akteuren auf globaler und lokal-regionaler Ebene sowie aus den konstitutiven strukturellen Prozessen in Wirtschaft, Gesellschaft und Staat.

In der als agro-industriell zu bezeichnenden Entwicklungsdynamik übernehmen globale Akteure im Wesentlichen die Steuerung lokal-regionaler Prozesse. In der Landwirtschaft dominieren standardisierte Massenprodukte, die von Billiglohnarbeitern herge- stellt werden und für den Massenkonsum vor allem auf globaler Ebene bestimmt sind. Rohstoff e und landwirtschaftliche Produkte des ländlich-peripheren Raumes warden abgezogen von den großen Zentren weltweit, so dass die Produktionslogik den globalen Märkten folgt. Ein Großteil der ländlichen Bevölkerung wird aus diesen Wirtschaftskreisläufen ausgegrenzt und „fl üchtet“ - sofern möglich - in die international Migration. Der Staat zieht sich weitgehend aus der Steuerung wirtschaftlicher Prozesse zurück und überlässt den global players das Feld. Die Natur dient lediglich als Ressourcenquelle und wird dementsprechend ausgebeutet. Beispiele in Lateinamerika sind dafür vor allem der Sojaanbau, die Herstellung von Biotreibstoff en und die Grundnahrungsmittelproduktion. Der ländliche Raum - Wirtschaft, Gesellschaft und Natur - wird in der agro-industriellen Entwicklungsdynamik im Wesentlichen als Produktionsraum genutzt.

Abb. 3: Entwicklungsdynamiken in ländlichen Räumen Lateinamerikas 


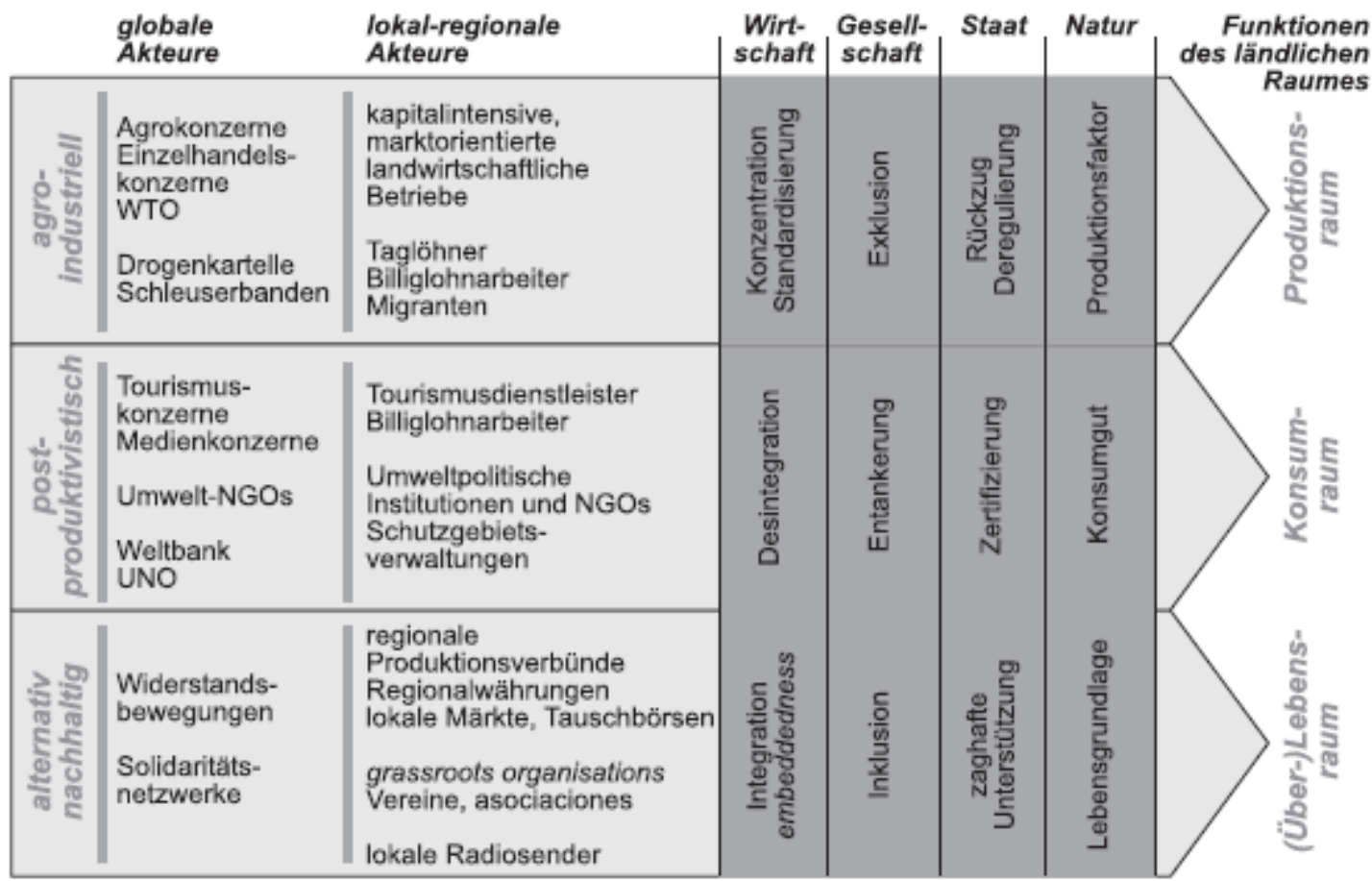

Entwurf: Martina Neuburger 2008.

Für die Durchsetzung des so genannten post-produktivistischen Entwicklungsmodells sind in ähnlicher Form globale Akteure die wichtigsten Entscheidungsträger. Hier fungiert der ländliche Raum jedoch als Konsumraum einer städtischen oder/und globalen Elite, die ihre Freizeit dort gestalten will und ökologische Ausgleichsfl ächen für die ,eigene“ degradierte Umwelt benötigt. Hier spielen vor allem Tourismuskonzerne, aber auch Umweltorganisationen, internationale Entwicklungsagenturen und Medien eine wichtige Rolle, denn sie fördern Umwelt- und Naturschutz oder verleihen Zertifi kate bzw. erarbeiten entsprechende Konventionen. Die lokal-regionale Bevölkerung, die den ländlichen Raum zur Produktion und als Lebensgrundlage benötigt, wird in ihren Nutzungsmöglichkeiten eingeschränkt oder muss sich den Schutz- und Nachhaltigkeitsideologien der globalen Akteure unterordnen. Naturnahe Flächen mit als schützenswert rachteten Ökosystemen können hingegen expandieren. Beispiele dafür sind in Lateinamerika vor allem in den Regionen des Ökotourismus zu fi nden. Aber auch in Gebieten der Rentner- und Zweitwohnsitze sowie in Naturschutzgebieten verschiedenster Schutzkategorien oder im Bereich von ökologisch bzw. regionstypisch zertifi -zierten landwirtschaftlichen Produkten greift diese Strukturierung.

In der als alternativ bezeichneten Entwicklungsdynamik schließlich dominieren eindeutig die lokal-regionalen Akteure, deren Hauptaugenmerk auf der Überlebenssicherung der lokalen Bevölkerung, auf der Sicherung der ökologischen Ressourcen und auf der Erhaltung der lokalen Gemeinschaft liegt. Die dazugehörigen lokalen Zusammenhänge und Wirtschaftskreisläufe lassen nur in sehr begrenztem Umfang Verknüpfungen zu übergeordneten Ebenen $\mathrm{zu}$ und beschränken sich im Wesentlichen auf international organisierte Solidaritätsnetzwerke und Unterstützergruppen von lokalen Widerstandsbewegungen. Die betreff enden Gruppen klinken sich teils bewusst aus den so genannten konventionellen Märkten aus und bauen Parallelstrukturen auf, die auf Solidarität, Vertrauen und Gerechtigkeit basieren. Teils werden sie jedoch im Zuge von Fragmentierungsprozessen aus diesen sehr viel größeren 
Märkten ausgegrenzt und sind von Armut, Marginalität und Illegalität betroff en. Die Entstehung von grassroots organisations, von lokalen Vereinen und Assoziationen, das Aufkommen lokaler Radiosender, von lokalen Märkten, Regionalwährungen und Tauschbörsen sind als Gegenbewegungen dazu Ausdruck dieses Entwicklungsmodells. Der ländliche Raum bildet in diesem Kontext den Lebens- und Überlebensraum für die lokale Bevölkerung.

Die dargestellten drei Entwicklungsmodelle, die sich in den zuvor beschriebenen Globalisierungseinfl üssen und lokalen Reaktionen in Lateinamerika auf vielfältige Weise widerspiegeln, überlagern sich in den ländlichen Gebieten sowohl zeitlich als auch räumlich. Diese Gleichzeitigkeit sehr unterschiedlicher Entwicklungsdynamiken bildet seit einigen Jahren die größte Herausforderung für die politischen Entscheidungsträger, denn bisherige planerische Maßnahmen, die von einer zwar regional diff erenzierten, jedoch innerhalb der Regionen vergleichsweise einheitlichen Strukturierung ausgingen, greifen damit ins Leere. Bislang konzentrieren sich staatliche Planungen für den ländlichen Raum fast ausschließlich auf die Landwirtschaft. Wie in den vorhergehenden Kapiteln gezeigt, hat sich der ländliche Raum aber wirtschaftlich und gesellschaftlich in den letzten Jahrzehnten immer stärker ausdiff erenziert, so dass im Zuge der Diskussionen um die Nueva Ruralidad eine Abkehr von sektoralen, agrarisch orientierten Planungskonzepten gefordert wird. In den neueren, weitgehend auf die Wissenschaft beschränkten Auseinandersetzungen um den ländlichen Raum in

Lateinamerika wurde erkannt, dass die zunehmende Heterogenität ländlicher Ökonomien und die Einkommensvielfalt ländlicher Haushalte konstitutive Elemente dieser neu zu defi nierenden Raumkategorie sind. Entsprechend sollten - so die involvierten Wissenschaftler - die institutionellen Rahmenbedingungen angepasst und neue, auch privatwirtschaftliche Vertragsformen etabliert werden. Auch die Aufrechterhaltung der Dichotomie zwischen Stadt und Land als planerische Raumkategorien wird für wenig zeitgemäß gehalten und die Berücksichtigung der Komplexität des ländlichen Wirkungsgefüges Ökosystem-AgroökosystemProduktionssystem oder Produktion- Ökomanagement-Armut gefordert. Erste Ansätze zur Umsetzung dieser neuen Konzepte sind in Lateinamerika zwar bereits beobachtbar so zum Beispiel das Programa Nacional de Fortalecimento da Agricultura FamiliarProgramm in Brasilien oder in der Participación Popular in Bolivien. Es fehlen jedoch zahlreiche Ideen, die vor allem Fehlentwicklungen wie Ressourcenausbeutung, Billiglohnarbeit und ähnliches beheben zu könnten.

\section{LITERATUR}

Adams Jr., R. \& Page, J. (2005): Do International Migration and Remittances Reduce Poverty in Developing Countries? In: World Development, 33 (10), S. 1645-1669.

Bayardo, R. \& Lacarrieu, M. (Hrsg.)(2003): Globalización y Identidad Cultural. Buenos Aires.

BHPbilliton (2009): Resourcing the future. Annual Report 2009. In: http://www.bhpbilliton.com/home/investors/reports/Documents/annualReport2009.pdf (Stand: 15.05.2010).

Branford, S. \& Rocha, J. (2002): Cutting the Wire. Th e Story of the Landless Movement in Brazil. London. 
Budka, P. (2004): Indigene Widerstandsbewegungen im Kontext von Globalisierung und Informations-und Kommunikationstechnologien. Das Fallbeispiel der EZLN in Mexiko. In: Journal für Entwicklungspolitik, 1, S. 33-44.

Calcagnotto, G. \& Nolte, D. (2000): Das Treff en der südamerikanischen Präsidenten in Brasília: Markstein der Integration oder Show-Veranstaltung einer aufkommenden Regionalmacht? In: Brennpunkt Lateinamerika, (17), S. 181-188.

Cleary, E. \& Steigenga, T. (Hrsg.)(2004): Resurgent Voices in Latin America. Indigenous Peoples, Political Mobilization, and Religious Change. New Brunswick, London.

Coy, M. \& Töpfer, T. (2009): Handel mit mineralischen Rohstoff en. Entwicklung mit Zukunft in Südamerika? In: Geographische Rundschau, 61 (11), S. 12-18.

Dehn, S. (2009): Geographien des Widerstands. Politisch-geographische Analyse der sozialen Bewegung APPO in Oaxaca, Mexiko. Tübingen (unveröff entlichte Diplomarbeit).

Gabbert, W. (2004): Der Aufstand der Zapatisten in Chiapas 1994. Vorbedingungen und Folgen. In: Bernecker, W.L. \& Braig, M. \& Hölz, K. \& Zimmermann, K. (Hrsg.): Mexiko heute - Politik, Wirtschaft, Kultur. 3. Aufl . Frankfurt am Main. S. 363-384.

Guanziroli, C. E. (2007): PRONAF Dez Anos Depois: Resultados e Perspectivas para o Desenvolvimento Rural. In: Revista de Economia e Sociologia Rural, 45, (2) S. 301328.

Hall, A. (2006): From Fome Zero to Bolsa Família: Social Policies and Poverty Alleviation under Lula. In: Journal of Latin American Studies, 38, S. 689-709.

Hees, W. \& Müller, O. \& Schüth, M. (Hrsg.)(2007): Volle Tanks - leere Teller. Der Preis für Agrarkraftstoff e: Hunger, Vertreibung, Umweltzerstörung. caritas international - brennpunkte 2007. Freiburg im Breisgau.

Hoefl e, S.W. (2004): Participação Política e Construção de Comunidade na Amazônia Central. In: Análise Social, 38 (169), S. 1091-1121.

Hoff mann, B. \& Schulz, M. (Hrsg.)(2002): Internet und Politik in Lateinamerika. Frankfurt am Main.

Husa, K. \& Parnreiter, C. \& Stacher, I. (Hrsg.) (2000): Internationale Migration. Die globale Herausforderung des 21. Jahrhunderts? Historische Sozialkunde, 17 Internationale Entwicklung. Wien.

INRA (Instituto Nacional de Reforma Agraria) (2010): Breve Historia del Reparto de Tierras en Bolivia. Resultados de la Reconducción Comunitaria de la Reforma Agraria en Bolivia, 1. La Paz.

Jerger, N. (2008): Ein Land in Bewegung. Transnationale Migration am Beispiel Bolivien - Spanien. Tübingen (unveröff entlichte Diplomarbeit). 
Jacobson, M. (2005): Die Expansion des transgenen Sojaanbaus und die Folgen für die Kleinbauern in der Provinz Santiago del Estero, Argentinien. In: Kohlhepp, G. (Hrsg.): Wirtschafts- und sozialräumliche Strukturwandlungen und Interessenkonfl ikte in Lateinamerika. Tübinger Geographische Studien, 142. Tübingen. S. 13-41.

Karnopp, E. (2006): Kleinbauern zwischen konventioneller und ökologischer Landwirtschaft. Das Beispiel der Region Vale do Rio Pardo (Brasilien). Tübinger Geographische Studien, 140. Tübingen.

Kohlhepp, G. (1998): Das internationale Pilotprogramm zum Schutz der tropischen Regenwälder Brasiliens. Globale, nationale, regionale und lokale Akteure auf dem Weg zu einer Strategie der nachhaltigen Entwicklung? In: Kohlhepp, G. \& Coy, M. (Hrsg.): Mensch-Umwelt-Beziehungen und nachhaltige Entwicklung in der Dritten Welt. Tübinger Geographische Studien, 119. Tübingen. S. 51-86.

Kohlhepp, G. (2008): Die Bedeutung Brasiliens in der modernen Biokraftstoff produktion. Zur Analyse der aktuellen Situation der Herstellung von Ethanol und Biodiesel. In: Martius-Staden-Jahrbuch, 55, S. 43-71.

Lander, E. (2008): Venezuela: Logros y Tensiones en los Primeros Ocho Años del Proceso de Cambio.

In: Stolowicz, B. (Hrsg.): Gobiernos de Izquierda en América Latina. Un Balance Político. Bogotá. S. 39-75.

Mançano Fernandes, B. (Hrsg.)(2008): Campesinato e Agronegócio na América Latina: A Questão Agrária Atual. São Paulo.

Marsden, T. (2003): Th e Condition of Rural Sustainability. Wageningen.

Marshall, A. (2009): S'Approprier le Désert. Agriculture Mondialisée et Dynamiques Socio-environnementales sur le Piémont Côtier du Pérou. Le Cas des Oasis de Virú et d'Ica-Villacuri. Paris (unveröff entlichte Dissertation).

Moldiz Mercado, H. (2008): Bolivia: Crisis Estatal y Proceso de Transformación. In: Stolowicz, B. (Hrsg.): Gobiernos de Izquierda en América Latina. Un Balance Político. Bogotá. S. 155-196.

Moyo, S. \& Yeros, P. (Hrsg.) (2005): Reclaiming the Land. Th e Resurgence of Rural Movements in Africa, Asia and Latin America. London, New York.

Neuburger, M. (2006): Property Rights de las Mujeres en el Área Rural de Brasil. Asociaciones de Mujeres en Mato Grosso entre la Pobreza y la Actividad Comunitaria. In: Molina, I. (Hrsg.): Rompiendo Barreras. Género y Espacio en el Campo y la Ciudad. Santiago de Chile, S. 167-185.

Neuburger, M. (2007): Lokal verankerte Armut in einer globalisierten Welt. Beispiele aus ländlichen Räumen Lateinamerikas. In: Mitteilungen der Österreichischen Geographischen Gesellschaft, 149, S. 217-240. 
Neuburger, M. (2008a): Globalisierung der ländlichen Räume in Lateinamerika. Chance oder Risiko für die Ernährungssicherung? In: Mitteilungen der Geographischen Gesellschaft in München, 90, S. 61-79.

Neuburger, M. (2008b): Global Discourses and the Local Impacts in Amazonia. Inclusion and Exclusion Processes in the Rio Negro Region. In: Erdkunde, 62 (4), S. 339-356.

Neuburger, M. (2010): Political Reforms and Local Governance in the Bolivian Amazon. In: van Lindert, P. \& Verkoren, O. (Hrsg.): Decentralized Development in Latin America. Experiences in Local Governance and Local Development. GeoJournal Library, 97. New York, Heidelberg, S. 87-99.

Neuburger, M. \& Coy, M. (2002): Chancen und Grenzen nachhaltiger Regionalentwicklung. Das Fallbeispiel des brasilianischen Amazonien. In: Geographische Rundschau, 54 (11), S. 12-20.

Nijenhuis, G. (2010): Th e Impact of Decentralisation on Local Development: Th e Case of Bolivia. In: van Lindert, P. \& Verkoren, O. (Hrsg.): Decentralized Development in Latin America. Experiences in Local Governance and Local Development. GeoJournal Library, 97. New York, Heidelberg, S. 69-86.

OLCA (Observatorio Latinoamericano de Confl ictos Ambientales) (2010): Área de seguimiento a las

actividades mineras. In: http://olca.cl/oca/mineras.htm (Stand: 15.05.2010)

Parker, C \& Mitchell, A. \& Trivedi, M. \& Mardas, M. (2009): Th e Little REDD+ Book. Global Canopy Programme. Oxford.

Reboratti, C.E. (2005): Efectos Sociales de los Cambios en la Agricultura. In: Ciencia Hoy, 15 (87), S. 52-61.

Redford, K.H. \& Fearn, E. (Hrsg.)(2007): Protected Areas and Human Displacement: A Conservation Perspective. WCS Working Paper, 29. New York.

Rio Tinto (2010): Annual Report 2010. In: http://www.riotinto.com/ourproducts.asp (Stand: 15.05.2010)

Rodriguéz Vignoli, J. (2004): Migración Interna en América Latina y el Caribe: Estudio Regional del Período 1980-2000. Serie Población y Desarrollo, 50. Santiago de Chile (CEPAL).

Röper, M. (2001): Planung und Einrichtung von Naturschutzgebieten aus sozialgeographischer Perspektive. Fallbeispiele aus der Pantanal-Region (Brasilien). Tübinger Geographische Studien, 134. Tübingen.

Schmidt, L. (2009): REDD from an Integrated Perspective. Considering Overall Climate Change Mitigation, Biodiversity Conservation and Equity Issues. DIEDiscussion Paper, 4/2009. Bonn. 
Simonis, U. (Hrsg.)(1996): Weltumweltpolitik. Grundriß und Bausteine eines neuen Politikfeldes. Berlin.

Sol Meliá (2005): Annual Report 2005. In: http://www.solmelia.com (April 2010).

Solimano, A. \& Allendes, C. (2007): Migraciones Internacionales, Remesas y el Desarrollo Económico: la Experiencia Latinoamericana. Serie Macroeconomía del Desarrollo, 59. Santiago de Chile (CEPAL).

Stolowicz, B. (Hrsg.)(2008): Gobiernos de Izquierda en América Latina. Un Balance Político. Bogotá.

Svampa, M. \& Antonelli, A.M. (Hrsg.) (2009): Minería Transnacional, Narrativas del Desarrollo y Resistencias Sociales. Buenos Aires.

UN (United Nations) (2009): International Migration Report 2006: A Global Assessment. ESA/P/WP.209. New York (Department of Economic and Social Aff airs).

Vale (2008): Sustainability Report 2008. In: http://www.vale.com/enus/investidores/relatorios-anuaise-de-sustentabilidade/pages/default.aspx ） (Stand: 15.05.2010).

Vono de Vilhena, D. (2006): Vinculación de los Emigrados Latinoamericanos y Caribeños con su País de Origen: Transnacionalismo y Políticas Públicas. Serie Población y Desarrollo, 71. Santiago de Chile (CEPAL).

Voth, A. (2002): Bewässerung und Obstanbau in Nordost-Brasilien - Neue Dynamik in einer Problemregion. In: Geographische Rundschau, 54 (11), S. 28-35.

World Bank (2007): World Development Report 2008: Agriculture for Development. Washington, DC. 